\title{
Vitrectomy with Inverted Internal Limiting Membrane Flap Technique for Treatment of a Traumatic Macular Hole
}

\author{
Emad Abdel Aal Saliem \\ Ophthalmology Department, Al-Azhar Faculty of Medicine, Assiut, Egypt \\ Corresponding author: Emad Saliem, Email: emadophtha@yahoo.com
}

\begin{abstract}
Background: Macular hole is a round full-thickness opening in foveal center which is an important cause of central vision loss. In most cases it is idiopathic, i.e., due to abnormal vitreo-foveal traction without any apparent predisposing conditions. Other causes include 1) High myopia. 2) Blunt ocular trauma.

Aim: to evaluate the anatomical closure rates and visual outcomes in patients with traumatic macular holes treated by vitrectomy with inverted internal limiting membrane (ILM) flap technique.

Patients and Methods: a prospective interventional study was conducted on 10 eyes of 10 patients with traumatic macular holes not spontaneously closed for more than 3 months after trauma and treated by vitrectomy with inverted internal limiting membrane flap technique at I-vision private center, Cairo and Alforsan eye private center, Assiut between September 2015 and December 2018. After surgery, both closure rate and visual outcome were re-evaluated monthly during the follow up period up to 6 months.

Results: the main best-corrected visual acuity improved after PPV with inverted ILM flap technique from 0.87 $\pm 0.09 \log$ MAR at baseline to $0.73 \pm 0.11 \log$ MAR at $1^{\text {st }}$ month, to $0.49 \pm 0.13 \log$ MAR at $3^{\text {rd }}$ month and reached to the maximum improvement $0.29 \pm 0.10 \log$ MAR by the end of $6^{\text {th }}$ month $(\mathrm{P}<0.001)$. The macular holes were successfully closed in all cases (100\%). Conclusion: vitrectomy with inverted internal limiting membrane flap technique is associated with complete closure of the macular hole with restoration of the normal architecture and also a significant improvement of vision.
\end{abstract}

Keywords: Vitrectomy, Inverted Internal Limiting Membrane Flap Technique, Traumatic Macular Hole.

\section{INTRODUCTION}

Full-thickness macular hole is a relatively common cause of loss of central vision. The pathogenesis in blunt trauma is not definitely known; some authors theorized that the macular rupture as a result of ocular trauma could result in rupture of the fovea ${ }^{(\mathbf{1})}$. Another hypothesis is that sudden separation of vitreous might be the cause of TMH, although many cases actually have an attached posterior vitreous ${ }^{(2)}$.

Optical coherence tomography (OCT) allowed the detection of changes in the subclinical inner retinal layers that could play a role in the pathogenesis of TMH formation ${ }^{(3)}$.

The standard treatment of $\mathrm{MH}$ is vitrectomy with internal limiting membrane (ILM) peeling, gas tamponade, and postoperative face-down positioning, aiming to completely eliminating the macular tractions and thus allowing the hole edges to come together ${ }^{(\mathbf{4 , 5})}$. In addition to elimination of tangential traction on the fovea, ILM peeling is believed to increase the extensibility of the retina and enhancing Müller cell gliosis which is associated with bridging the gap between the retinal hole edges ${ }^{(6,7)}$.

The $\mathrm{MH}$ is closed by this standard procedure in approximately $90 \%$ of cases $^{(8)}$. However, in some cases the standard procedure does not successfully close the MH, such as MHs with large diameters, those have existed for a long time, and secondary MHs resulting from uveitis, ocular trauma, high myopia, and proliferative vitreoretinopathies ${ }^{(\mathbf{9 , 1 0})}$. Up to that time, there was no effective treatment for these MHs.
Recently, Michalewska et al. ${ }^{\left({ }^{9}\right)}$ have reported the efficacy of the inverted ILM flap technique for large macular holes. Kuriyama ${ }^{(11)}$ and Michalewska et al. (12) also found that the inverted ILM flap technique contributed to a high macular hole closure rate in highly myopic eyes. The present study is the first one that designed to evaluate the surgical and visual outcomes of the vitrectomy with inverted ILM flap technique as a line of treatment of non-spontaneously closed TMHs for more than 3 months.

Aim: to evaluate the anatomical closure rates and visual outcomes in patients with traumatic macular holes treated by vitrectomy with inverted internal limiting membrane flap technique.

\section{PATIENTS and METHODS}

This prospective interventional study included 10 eyes of 10 patients $(7$ males and 3 females), with traumatic macular holes documented by FFA and OCT who had underwent pars plana vitrectomy with inverted internal limiting membrane flap technique at I-vision private center, Cairo and Alforsan eye private center, Assiut, between September 2015 and December 2018. The patients' ages ranged from $21 \mathrm{y}_{\mathrm{s}}$ to $45 \mathrm{y}_{\mathrm{s}}$ with the main of $33.8 \pm 7.91 y_{s}$. The main of duration of symptoms was $15.5 \pm 2.07 y$ s. An informed written consent was taken from each participant in the study. The study was approved by the Ethics Board of Al-Azhar University. 


\section{Patients' selection}

Inclusion criteria: traumatic MHs not spontaneously closed for more than 3 months according to OCT findings.

Exclusion criteria included:

1- Patient having pseudo-macular hole.

2- Other causes of macular holes.

3- Recurrent macular hole.

4- Other trauma associated ocular complications.

\section{Preoperative evaluation:}

All the patients were subjected to completeo preoperative ophthalmic assessment; Detailed historyo

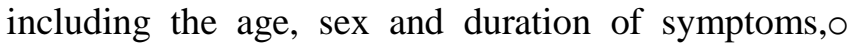
uncorrected (UCVA) and best corrected visual acuityo (BCVA), slit lamp examination, indirecto ophthalmoscopy, in addition A- and B-ultrasound scanning, fundus fluorescein angiography (FFA), ando optical coherence tomography (OCT) were done to all patients. All eyes were phakic before surgery.

\section{Surgical procedure:}

All the patients underwent $23 \mathrm{G}$ three-port pars plana vitrectomy. Following core vitrectomy, triamcinolone acetonide was used intraoperatively to facilitate the visualization of the vitreous and posterior hyaloid in all cases. The removal of ILM is considered to be a contributing factor in the success of macular hole surgeries, so, a brilliant blue dye assisted ILM peeling was done in a circular fashion around the macular hole making it easier to be visualized and manipulated. In contrast to the conventional ILM peeling, in this technique the ILM is not completely removed from the retina but is left in place, attached to the edges of the $\mathrm{MH}$ and inverted into the hole after trimming with cutter. Care should be taken not to include the deeper layers in the forceps' grasp, which may further damage the surrounding retinal tissues. To stabilize the ILM flap, sodium hyaluronate was placed on the inverted ILM. By the end of surgery, fluid-gas exchange by sulpherhexachloride (SF6) was used as gas tamponade. Patients remained in the face-down position for 5-7 days postoperatively. Primary outcome measures were complete closure of the macular hole with restoration of the normal macular architecture and improvement of vision.

\section{Postoperative follow up:}

All the patients were followed up for 6 months postoperatively. Follow up was scheduled as follows: 1st day, 1st week, 1st month, 3rd month and $6^{\text {th }}$ month. In each visit the followings items were assessed.

Best corrected visual acuity.

Corneal clarity and ocular complications.

Assessment of IOP by applanation tonometry.

Fundus examination.

OCT images were also taken to establish the closure of macular hole.

\section{Statistical analysis}

Statistical analyses were performed using SPSS version 22 (IBM Corporation, Chicago, IL, USA). The best-corrected visual acuity values were converted into LogMAR for statistical analysis. Mean, standard deviation and range were estimated. It was assessed with the analysis of variance (ANOVA) test followed by LSD test. P Value was considered significant as the following: $\mathrm{P}<0.05$ means it is statistically significant, $\mathrm{P}<0.001$ means it is statistically highly significant.

\section{RESULTS}

The study included 10 eyes of 10 patients ( 7 males and 3 females) with traumatic macular hole who had underwent vitrectomy with inverted ILM flap technique with brilliant blue dye enhancement and gas injection from September 2015 to December

2018. The demographic data of these patients are shown in Table 1.

Table (1): The demographic data of patients included in the study.

\begin{tabular}{|c|l|c|}
\hline \multirow{2}{*}{ Age of patients in year } & Range & $21-45 \mathrm{y}_{\mathrm{s}}$ \\
\cline { 2 - 3 } Gender & Mean \pm SD & $33.8 \pm 7.91$ \\
\hline \multirow{2}{*}{ Affected eye } & Male & 7 \\
\cline { 2 - 3 } & Female & 3 \\
\hline Lens status & Rt & 4 \\
\hline Preoperative BCVA/LogMAR & Lt & 6 \\
\hline unit & Phakic & 10 \\
\hline Postoperative BCVA/LogMAR & Mean \pm SD & $0.87 \pm 0.09$ \\
\cline { 2 - 3 } unit & Range & $(0.70-1.00)$ \\
\hline Hole status based on OCT & Range & $0.50 \pm 0.21$ \\
\hline & Closed & $(0.20-0.90)$ \\
\cline { 2 - 3 } & Not closed & 10 \\
\hline
\end{tabular}

The main best-corrected visual acuity improved from $0.87 \pm 0.09 \log$ MAR at baseline to $0.73 \pm 0.11$ $\log$ MAR at $1^{\text {st }}$ month followed by $0.49 \pm 0.13 \log$ MAR at $3^{\text {rd }}$ month and reached to the maximum improvement 
$0.29 \pm 0.10 \log$ MAR by the end of $6^{\text {th }}$ month which is considered statistically highly significant $(\mathrm{P}<0.001)$ (Table 2 and Figure 1).

Table (2): The main best-corrected visual acuity preoperative and postoperative at $1^{\text {st }}, 3^{\text {rd }}, 6^{\text {th }}$ months after vitrectomy with inverted ILM flap technique for TMH.

\begin{tabular}{|c|c|c|c|}
\hline & \multirow{2}{*}{$\begin{array}{c}\text { Mean } \pm \text { SD } \\
\text { (Range) }\end{array}$} & \multicolumn{2}{|c|}{ 95\% Confidence Interval for Mean } \\
\hline & & Lower Bound & Upper Bound \\
\hline Baseline & $\begin{array}{l}0.87 \pm 0.09^{\mathrm{a}} \\
(0.70-1.00)\end{array}$ & 0.80 & 0.94 \\
\hline First month & $\begin{array}{l}0.73 \pm 0.11^{\mathrm{b}} \\
(0.60-0.90)\end{array}$ & 0.65 & 0.81 \\
\hline Third month & $\begin{array}{l}0.49 \pm 0.13^{c} \\
(0.30-0.70)\end{array}$ & 0.40 & 0.58 \\
\hline Sixth month & $\begin{array}{l}0.29 \pm 0.10^{\mathrm{d}} \\
(0.20-0.50)\end{array}$ & 0.22 & 0.36 \\
\hline
\end{tabular}

* values are expressed as mean \pm SD. ANOVA followed by LSD's multiple range tests.

* values in column with different alphabetic characters are statistically significant at $\mathrm{P}<0.05$.

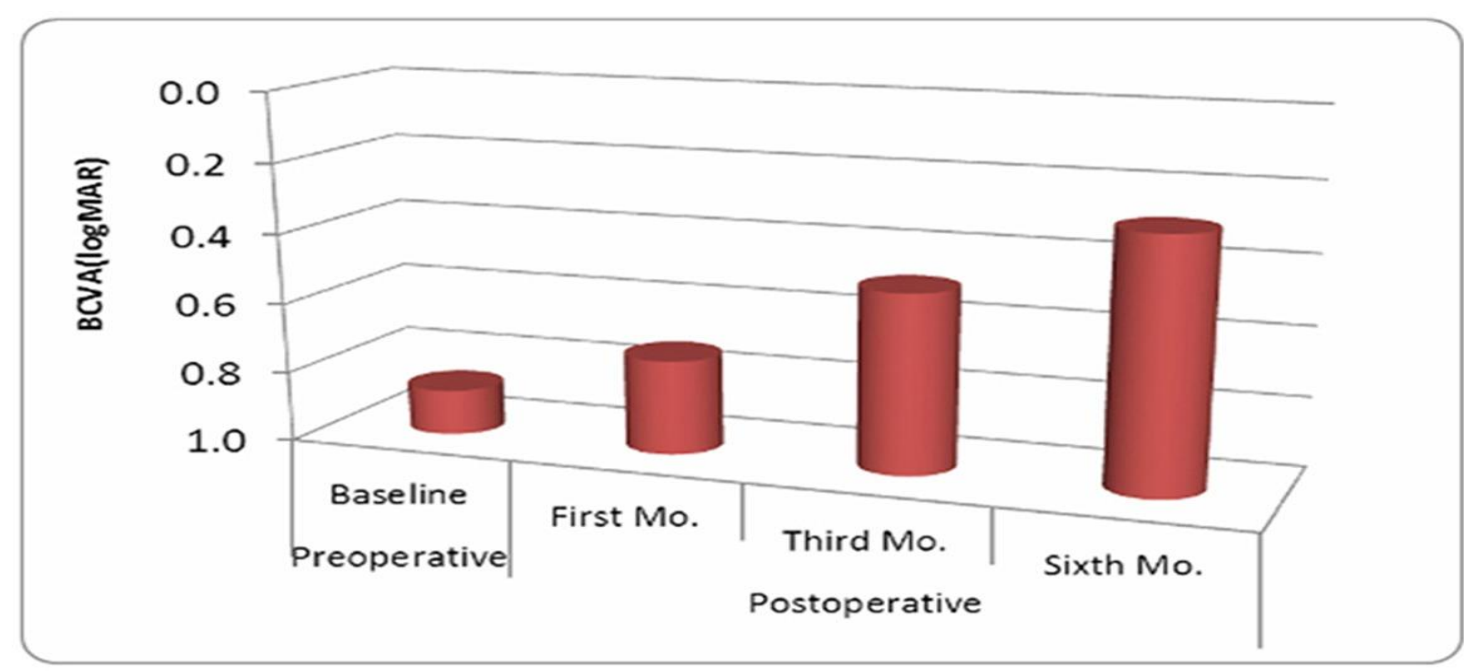

Figure (1): The main best-corrected visual acuity preoperative and postoperative at $1^{\text {st }}, 3^{\text {rd }}, 6^{\text {th }}$ months after vitrectomy with inverted ILM flap technique for TMH.

One month after surgery, the BCVA remained unstable, started to be sufficiently stable after three months $(\mathrm{p}<0.05)$ with marked improvement at the $6^{\text {th }}$ month after the operation which is considered statistically highly significant $(\mathrm{p}<0.001)$.

The primary macular hole closure rate was $100 \%$ and successful hole closure was confirmed by ophthalmoscopy which demonstrated disappearance of the hole and restoration of the foveal reflex and also by OCT which demonstrated restoration of normal foveal architecture during follow-up where the ILM acts like a scaffold for tissue proliferation enhancing the photoreceptors to assume new positions in direct proximity to the fovea and perfect anatomic restoration occurs.

No evidence of intra-operative or postoperative complications as retinal detachment, vitreous hemorrhage, or elevated intraocular pressure. Localized subconjunctival hemorrhage may be noticed in some cases postoperatively at the site of sclerotomies. 


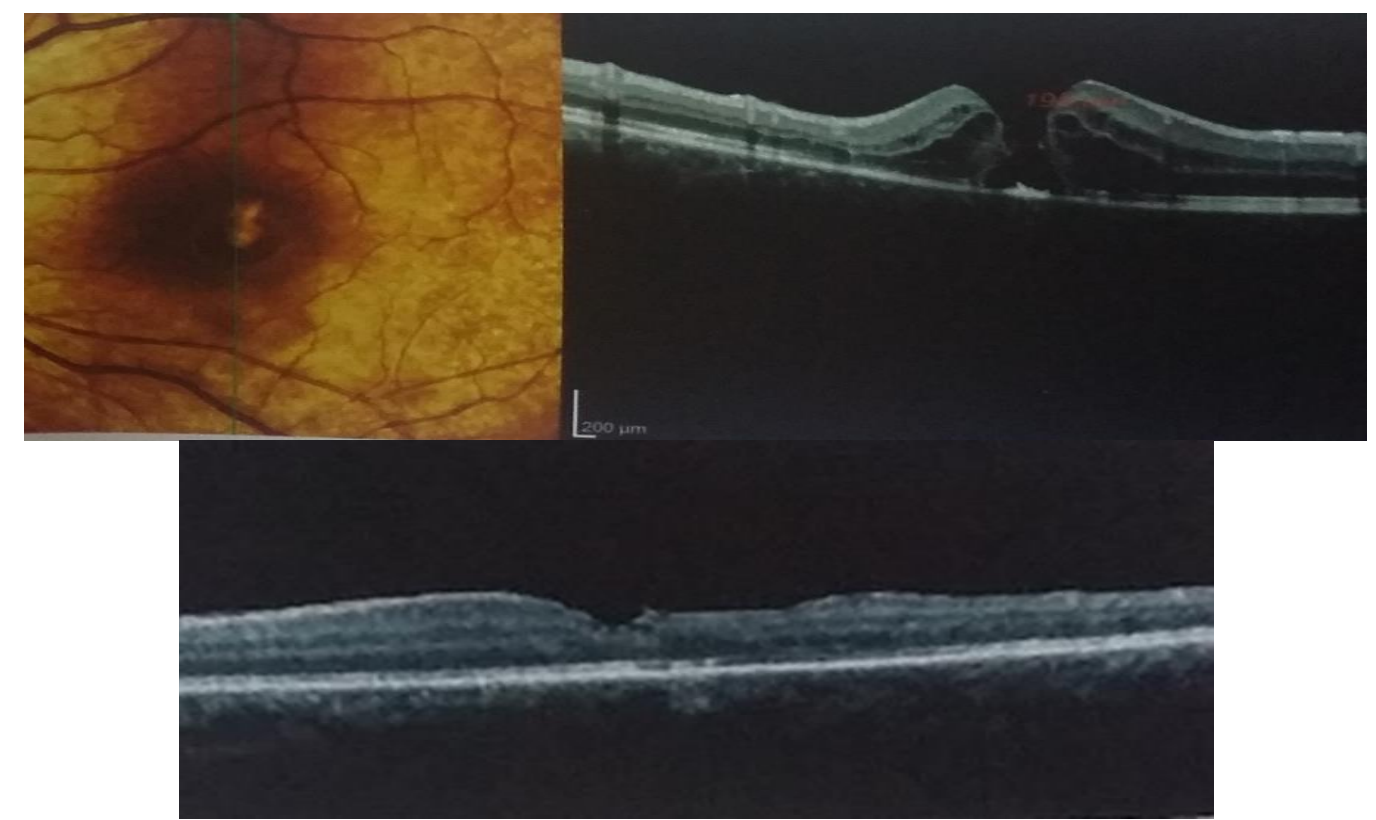

Figure 2: preoperative Color and OCT of a full thickness TMH in a male patient aged 21ys treated by PPV with inverted ILM flap technique (top) and 6 months postoperative OCT of the same patient revealed complete closure of the $\mathrm{MH}$ with restoration of the foveal contour with visible glial plug filling the MH defect (bottom).

\section{CASE NO. 2}

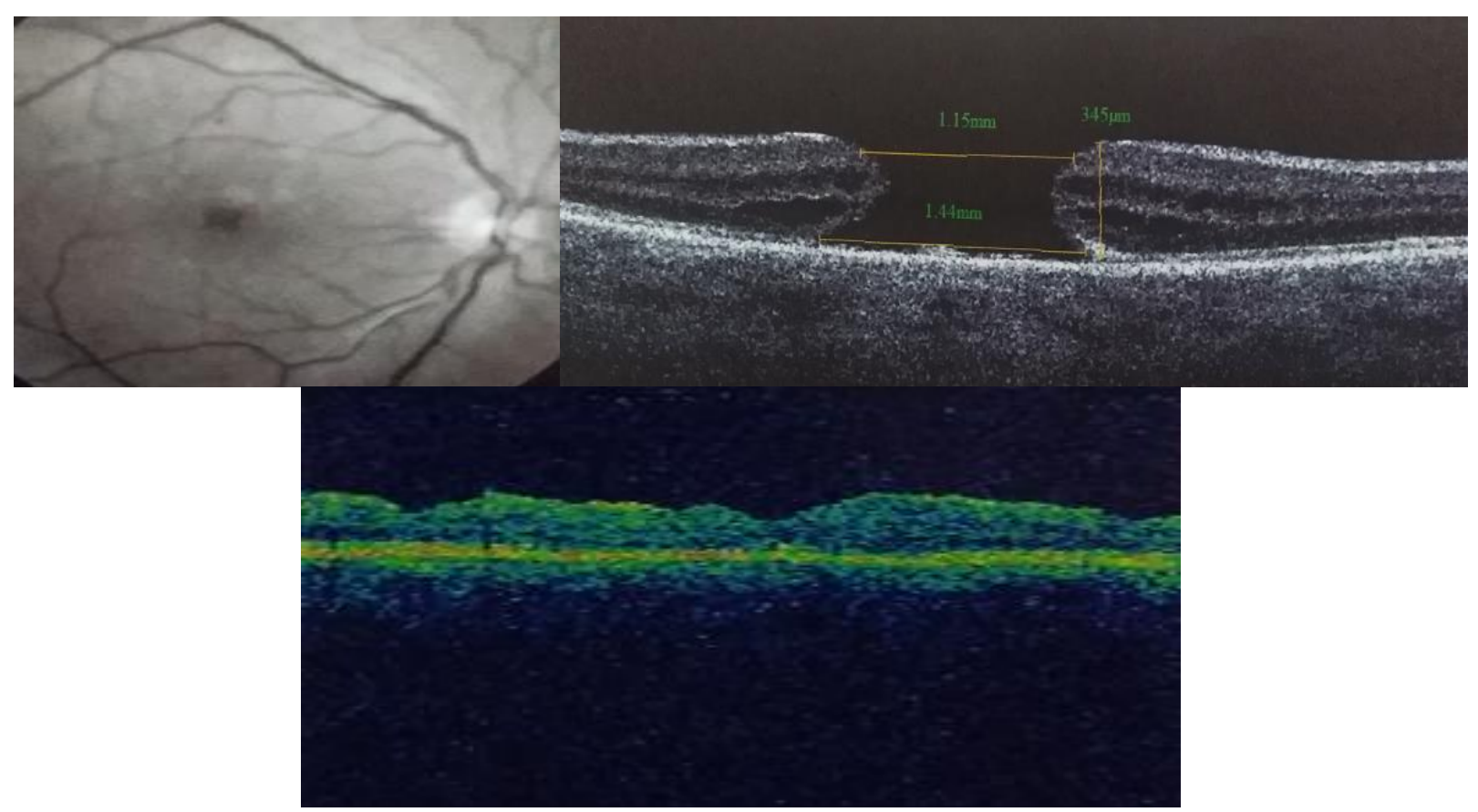

Figure 3: preoperative red free and OCT of a large full thickness TMH in a male patient aged 42ys (top)

treated by PPV with inverted ILM flap technique and 6 months postoperative OCT of the same patient revealed closure of $\mathrm{MH}$ with visible glial plug filling the defect and restoration of the foveal contour (bottom).

\section{DISCUSSION}


Ocular trauma is one of the most common causes of secondary MHs. Traumatic macular holes are thought to be caused by blunt trauma that results in a flattening of the globe in the anterior-posterior direction causing a stretching on the perpendicular plane inducing a significant impact force leading to development of a hole in the retina. In order to release the tangential forces acting on the macular hole, different techniques for macular hole surgery had been used. Vitrectomy with ILM peeling has the most positive effect on final outcome, in addition to promoting hole closure, ILM peeling also reduces the probability of its reopening ${ }^{(\mathbf{1 3}, 14)}$.

The inverted ILM flap technique was initially developed to improve the functional and anatomical results in cases with large MHs; then, the indications for this technique were expanded to include MHs caused by high myopia ${ }^{(\mathbf{1 0 , 1 2})}$.

In the present study, $\mathrm{i}$ chose to do the inverted ILM flap technique to treat TMHs not spontaneously closed for more than 3 months after trauma and the $\mathrm{MH}$ closure rate after this technique was significantly high.

In this technique, after core vitrectomy and staining of ILM with brilliant blue, ILM is not completely removed from the retina and left in place. This peeled-off ILM contains Müller cell fragments which can provoke gliosis both inside the retina and on the surface of the ILM. Also, the ILM may be a scaffold for tissue proliferation creating a microenvironment that encourages correct photoreceptor positioning and finally improving postoperative anatomical and functional outcomes.

To minimize iatrogenic trauma during ILM peeling, Michalewska et al $^{\left({ }^{(15)}\right.}$ introduced a new modified procedure in which ILM is only peeled from the temporal side of the fovea. They found that, this temporal inverted ILM flap technique was as effective as the classic inverted ILM flap technique for the repair of large MHs.

Many authors ${ }^{(\mathbf{1 5}, 16)}$ have reported similar satisfactory results with the inverted flap technique for treating large idiopathic MHs, so, they expanding its use to involve myopic and recurrent MHs. They reported that the rate of complete closure was $100 \%$ for high-myopic MHs, and they confirmed that the process of foveal architectural repair after the surgery continued over at least a 12-month period and this technique allowed not only a high closure rate in myopic MHs, but also a functional improvement in visual acuity.

In a comparison of the inverted ILM flap technique and ILM peeling alone, Chen et al. ${ }^{(\mathbf{1 7})}$ reported that 23-gauge PPV with the inverted ILM flap technique contributed to better anatomical success. However, they found that there were no statistically significant differences in postoperative BCVA between the inverted ILM flap technique and ILM peeling.

In the present study 10 eyes of 10 patients with traumatic macular holes were treated by vitrectomy with inverted ILM flap technique and revealed that the primary macular hole closure rate was $100 \%$ with higher anatomical and functional success rates.

The main best-corrected visual acuity improved after PPV with inverted ILM flap technique from $0.87 \pm 0.09 \log$ MAR at baseline to $0.73 \pm 0.11 \log$ MAR at $1^{\text {st }}$ month, to $0.49 \pm 0.13$ $\log$ MAR at $3^{\text {rd }}$ month and reached to the maximum improvement $0.29 \pm 0.10 \log$ MAR by the end of $6^{\text {th }}$ month which is considered statistically highly significant $(\mathrm{P}<0.001)$.

The present study showed that a shorter duration of symptoms is associated with complete closure of the macular hole and greater visual improvement and these results agree with results of Kelly and Wendel ${ }^{(4)}$ that reported, the best functional and anatomical results were obtained when symptoms had been present for less than 6 months, also, the postoperative visual acuity depends in many respects on the duration of the hole.

In the present study, small sample size and short term follow up were considered a weakening points due to lack of cases of TMHs, in contrast to the point of strength of this study is that, it is the first one designed to evaluate the functional and anatomical outcomes of vitrectomy with the inverted ILM flap technique for treatment of nonspontaneously closed traumatic macular holes.

\section{CONCLUSION}

In short term follow up period, vitrectomy with the inverted ILM flap technique seems to be effective surgery for traumatic MHs, completely closes the macular hole and restores the normal macular architecture, thus improving both functional and anatomical outcomes.

\section{REFERENCES}

1. Takahashi M, Yanagiya N, Akiba J, Shimizu A, Kakehashi A, Kado M et al. (1996): Clinical characteristics of traumatic macular holes. Japn J Ophthalmol, 40(4): 544-547.

2. Johnson RN, McDonald HR, Lewis H, Grand MG, Murray TG, Mieler WF et al. (2001): Traumatic macular hole: observations, pathogenesis, and results of vitrectomy surgery. Ophthalmology, 108(5): 853-857.

3. Stalmans $P$ and Oehrens AM (2006): Optical coherence tomographic documentation of the formation of a 
traumatic macular hole. Am J Ophthalmol, 142(5): 866869.

4- Kelly NE and Wendel RT (1991): Vitreous surgery for idiopathic macular holes. Results of a pilot study. Arch Ophthalmol, 109: 654-659.

5- Parravano M, Giansanti F, Eandi CM, Yap YC, Rizzo S, Virgili G (2015): Vitrectomy for idiopathic macular hole. Cochrane Database Syst Rev., 5: CD009080.6. Yang SM, Choi YM, Oh J, Kim SW, Huh K (2013): Glial proliferation after vitrectomy for a macular hole: a spectral domain optical coherence tomography study. Graefes Arch Clin Exp Ophthalmol, 251: 477484.

7. Gregor ZJ (1996): Surgery for idiopathic full-thickness macular holes. Eye (Lond), 10:685-690.

8. Mester $\mathbf{V}$ and Kuhn $\mathbf{F}$ (2000): Internal limiting membrane removal in the management of full-thickness macular holes. Am J Ophthalmol., 129: 769-777.

9- Michalewska Z, Michalewska J, Adelman RA, Nawrocki J (2010): Inverted internal limitingmembrane flap technique for large macular holes. Ophthalmology, 117 :2018-2025.

10-Morizane Y, Shiraga F, Kimura $\mathrm{S}$ et al. (2014): Autologous transplantation of the internal limiting membrane for refractory macular holes. Am J Ophthalmol, 157: 861-869.

11- Kuriyama S, Hayashi H, Jingami Y, Kuramoto N, Akita J, Matsumoto M et al. (2013): Efficacy of internal limiting membrane flap technique for the treatment of macular hole in high myopia. Am J Ophthalmol., 156: 125-131.

12- Michalewska $Z$, Michalewska J, DulczewskaCichecha K, Nawrocki J (2014): Inverted internal limiting membrane flap technique for surgical repair of myopic macular holes. Retina, 34: 664-669.

13- Yamanishi S, Emi K, Motokura M, Oshima Y, Nakayama M, Watanabe M (2001): Visual outcome of macular hole surgery with internal limiting membrane peeling. Nihon Ganka Gakkai Zasshi, 105:788-793.

14-Park DW, Sipperley JO, Sneed SR, Dugel PU, Jacobsen J (1999): Macular hole surgery with internallimiting membrane peeling and intravitreous air. Ophthalmology, 106: 1392-1397.

15- Michalewska Z, Michalewski J, DulczewskaCichecka K, Adelman RA, Nawrocki J (2015):Temporal inverted internal limiting membrane flap technique versus classic inverted internal limiting membrane flap technique: a comparative study. Retina, 35(9): 1844-1850.

16- Hayashi $H$ and Kuriyama $S$ (2014): Foveal microstructure in macular holes surgically closed by inverted internal limiting membrane flap technique. Retina, 34(12): 2444- 2450.

17-Chen SN and Yang CM (2016): Inverted Internal limiting membrane insertion for macular hole-associated retinal detachment in high myopia. Am J Ophthalmol, 162: 99-106. 